excluded from this study. All patients underwent Tc-99m ECD SPECT and were classified by the number of positive antiphospholipid antibodies they carried. The heterogeneity of brain perfusion was defined as the coefficient of variation. Analysis of variance (ANOVA) was used to evaluate the differences between groups.

Results Total 60 adult patients were included in this study. There were 54 patients in the case group and 6 patients in the control group. The mean age was $38.3 \pm 11.5$ years. There were 52 women and 8 men. There was no significant difference in mean brain perfusion between groups $(p=0.69)$. However, Tc-99m ECD SPECT demonstrated significant heterogeneity of brain perfusion in relation to the number of antiphospholipid antibodies $(\mathrm{p}=0.01)$.

Conclusions This is the first study to show that Tc-99m ECD SPECT can detect the increased heterogeneity of brain circulation in non-criteria antiphospholipid antibody carriers with neuropsychiatric manifestations.

\section{THE ROLE OF INTRACISTERNAL DEPOSITED IGG IN THE PATHOGENESIS OF NEUROPSYCHIATRIC SYMPTOMS OF SYSTEMIC LUPUS ERYTHEMATOSUS}

C Xia, Y Guo, X Fang, W Qiao, GM Deng*. Nanjing Medical University, Key Laboratory of Antibody Techniques of Ministry of Health, Nanjing, China

\subsection{6/lupus-2017-000215.15}

Background and Aims Systemic lupus erythematosus (SLE) is a chronic autoimmune disease characterised by high levels of autoantibodies and multi-organ tissue damage including damage of the central system. Nerve system disease in SLE includes neurologic and psychiatric events, collectively termed neuropsychiatric SLE (NPSLE). Currently, the pathogenesis of NPSLE is unclear. We investigated pathogenesis of NPSLE in this study.

Methods To understand the pathogenesis of NPSLE, we analysed the medical records of 1131 patients with SLE, and conducted experiments by using lupus-prone mice and mice with intracisternal injections of lupus serum containing high level of autoantibody and mice with gene deficiency.

Results There are 59 patients with NPSLE clinical manifestation including headaches, seizure disorder, cognitive dysfunction, cerebrovascular disease, etc. MRI examination in 14 patients with NPSLE indicates 50\% normal and 50\% abnormal signals. Lupus-prone mice spontaneously develop meningitis. Meningitis was developed in normal mice with intracisternal injection of lupus serum but not healthy serum; $\mathrm{IgG}$ is a major contributor in this meningitis. Monocyte/macrophage, complement and selectin are involved in the development of meningitis induced by lupus serum. This meningitis is dependent on dose of $\operatorname{IgG}$ but not associated with kinds of autoantibodies and systemic disease activity. Severity of meningitis induced by lupus serum IgG was significantly reduced in TNF deficient mice compared to wild mice.

Conclusions The deposited IgG or IgG contained immune complex in brain tissue exerts an important role in the pathogenesis of meningitis. This finding will promote development of effective therapeutic strategy to patients with NPSLE.

\section{Parallel Session 2: Cell targeting in SLE}

\section{HIGH SALT PROMOTES SYSTEMIC LUPUS ERYTHEMATOSUS BY TET2-INDUCED DNA DEMETHYLATION AND DRIVING THE DIFFERENTIATION OF TFH CELLS}

${ }^{1} \mathrm{H}$ Wu*, ${ }^{1} \mathrm{X}$ Huang, ${ }^{1} \mathrm{H}$ Qiu, ${ }^{1} \mathrm{M}$ Zhao, ${ }^{1} \mathrm{Q}$ Lu. ${ }^{1}$ The Second Xiangya Hospital of Central South University, dermatology, Chang Sha, China

\subsection{6/lupus-2017-000215.16}

Background and Aims Systemic lupus erythematosus (SLE) is an autoimmune disorder that is characterised by the presence of autoantibodies and immune dysregulation. The pathogenesis of SLE has not been elucidated. The induction of epigenetic changes by environmental factors such as diet may also be relevant. A high-salt diet is considered an important contributor to cardiovascular and renal diseases, and recent research has indicated that a high-salt diet can induce autoimmunity.

Methods In this study, the effects of high salt on various immune cells and in MLR/lpr mice were observed, and the underlying mechanisms were investigated by flow cytometry, high-throughput sequencing, DNA methylation map, ChIPQPCR.

Results In this study, high salt (sodium chloride, $\mathrm{NaCl}$ ), under physiological conditions, was demonstrated to increase the differentiation of Tfh. A high-salt diet markedly increased lupus features in MRL/lpr mice. The mechanism is NaCl-induced DNA demethylation via the recruitment of the hydroxytransferase Ten-Eleven Translocation 2 (TET2). Gene silencing of TET2 obviously diminished $\mathrm{NaCl}$-induced $\mathrm{T}$ fh cell polarisation in vitro. In addition, the gene expression of $\operatorname{sh} 2 \mathrm{~d} 1 \mathrm{a}, \operatorname{map} 3 \mathrm{k} 1$, spn and stat $5 \mathrm{~b}$ was enhanced after $\mathrm{NaCl}$ treatment, consistent with the findings in lupus $\mathrm{CD}^{+} \mathrm{T}$ cells. However, only spn was directly regulated by TET2, and spn was not the sole target for $\mathrm{NaCl}$.

Conclusions High-salt treatment promotes SLE in mice and the underlying mechanism might be $\mathrm{NaCl}$ enhancing $\mathrm{Tfh}$ cell differentiation by TET2 inducing global and gene specific DNA demethylation. Our findings not only explain the epigenetic mechanisms of high-salt induced autoimmunity but also provide an attractive molecular target for intervention strategies of SLE.

\section{Parallel Session 4: Lupus reflections across the continents: are we addressing the needs of our patients?}

\section{CAPABILITIES OF EUROPEAN LUPUS GROUPS: MEMBERS OF LUPUS EUROPE}

K Lerstrøm. Farum, Denmark

10.1136/lupus-2017-000215.17

Background Lupus patient organizations(POs) are becoming increasingly important stakeholders in political and medical healthcare decision-making processes. LUPUS EUROPE is an umbrella organization of national lupus groups in Europe. Objectives To identify the different structures and capabilities among European lupus groups. 